\title{
Costo-efectividad de ceftazidima/avibactam versus colistin + meropenem en el tratamiento de infecciones por enterobacterias resistentes a carbapenémicos en Chile
}

\author{
Cost-effectiveness of ceftazidime/avibactam versus colistin + meropenem for treatment \\ of carbapenemic-resistant enterobacteria infections in Chile
}

\author{
Magda Gutiérrez A. y Cecil Fandiño
}

Los autores de este manuscrito declaran ser empleados de Pfizer Chile S.A. laboratorio fabricante del principio activo ceftazidima/avibactam

Este trabajo no presentó financiamiento institucional.

Recibido: 28 de agosto de 2020 / Aceptado: 12 de enero de 2021

\section{Resumen}

Introducción: Ceftazidima-avibactam (C/A), ha demostrado reducir las tasas de mortalidad y el riesgo de nefrotoxicidad, comparado con colistin, la terapia convencional. Objetivo: Estimar la costo-efectividad de $\mathrm{C} / \mathrm{A}$ versus colistin + meropenem en el tratamiento de infecciones por Enterobacteriaceae resistentes a carbapenémicos (ERC) en Chile. Material y Métodos: Se adaptó un modelo económico tipo árbol de decisión. Se utilizó la perspectiva del pagador público, un horizonte de tiempo de 30 días con extrapolación a la expectativa de vida. La información clínica se derivó de un estudio observacional. Los costos de los medicamentos y de atención corresponden a reportes locales. Los resultados se expresan como razón de costo-efectividad incremental (RCEI) por año de vida ganado (AVG) y por año de vida ajustado por calidad (AVAC) en pesos chilenos y en dólares estadounidenses (US\$ $1,00=\$ 792,2218$ ). Resultados: Se obtuvieron 8,65 y 6,48 AVGs y 6,44 y 4,27 AVACs, para C/A y colistin + meropenem, respectivamente. La RCEI estimada de C/A fue $\$ 940.488$ (US\$1.187,2) por AVG y $\$ 938.715$ (US\$1.184,9) por AVAC. Discusión: Dada la falta de publicaciones o evidencia, el modelo se basa en un estudio observacional. C/A reduciría la proporción de muertes e incrementaría los AVG y los AVAC, resultando en una alternativa costo-efectiva versus colistin + meropenem para ERC.

Palabras clave: ceftazidima/avibactam; Enterobacteriaceae resistente a carbapenémicos; infecciones bacterianas gramnegativas; análisis de costo-efectividad.

\begin{abstract}
Background: Ceftazidime-avibactam (C/A), has shown reduction in mortality rates and risk of nephrotoxicity, compared to colistin, conventional therapy. Aim: To estimate the cost-effectiveness of $\mathrm{C} / \mathrm{A}$ versus colistin + meropenem in the treatment of infections due to carbapenem-resistant Enterobacteriaceae (CRE) in Chile. Methods: An economic decision tree type model was adapted. The perspective of the public payer was used with a time horizon of 30 days and extrapolation to life expectancy. The clinical information was derived from an observational study. Medication and care costs correspond to local reports. The results are expressed as incremental cost-effectiveness ratio (ICER) per life year gained (LYG) and per quality adjusted life year (QALY) in Chilean pesos and US dollars (US\$ $1.00=\$ 792.2218$ ). Results: 8.65 and 6.48 LYGs and 6.44 and 4.27 QALYs were obtained, for C/A and colistin + meropenem, respectively. The estimated ICER for C/A was $\$ 940,488$ (US\$1,187.2) per AVG and $\$ 938,715$ (US\$1,184.9) per QALY. Discussion: Given the lack of publications or evidence, the model is based on an observational study. C/A would reduce the death rate and increase LYGs and QALYs, resulting in a cost-effective alternative vs. colistin + meropenem for CRE.

Keywords: avibactam/ceftazidime drug combination; carbapenemresistant Enterobacteriaceae; gramnegative bacterial infections; cost-benefit analysis.
\end{abstract}




\section{Introducción}

L a prevalencia de la resistencia a los antimicrobianos está aumentando en todo el mundo y representa una grave amenaza para la salud pública ${ }^{1}$. Cada año, al menos 2 millones de personas en los Estados Unidos de América (E.U.A.) desarrollan infecciones con bacterias que son resistentes a más de un grupo de antimicrobianos. En América Latina, entre 10 y $50 \%$ de las infecciones son causadas por bacterias resistentes a los antimicrobianos, dependiendo del tipo de microorganismo $^{2}$. En E.U.A. y Europa, cerca de 50.000 personas mueren como resultado directo de este tipo de resistencia y, en el mundo, se estima que la cifra podría superar las 700.000 muertes por año. Para 2050, 10 millones de personas podría morir como consecuencia directa de microorganismos multi-resistentes e incurrir acumulativamente en al menos $\$ 100$ billones de dólares en costos hospitalarios y pérdidas de productividad si no se encuentran soluciones pro-activas ahora para frenar el aumento de la resistencia a los medicamentos ${ }^{3}$.

Entre las bacterias resistentes más preocupantes se encuentran las Enterobacteriaceae resistentes a carbapenémicos (ERC) debido a que en infecciones invasoras (p. ej., infecciones del torrente sanguíneo) se han asociado con altas tasas de mortalidad (hasta 40 a $50 \%$ en algunos estudios $)^{2,4}$, a estancias hospitalarias prolongadas e incremento de los costos médicos ${ }^{5,6}$. La propagación mundial de las ERC es una amenaza importante para las poblaciones de pacientes vulnerables en todo el mundo. ${ }^{6}$ En Chile, un estudio realizado por el Grupo Colaborativo de Resistencia Bacteriana, sobre incidencia de bacterias multi-resistentes en 20 unidades de cuidado intensivo de adulto en el período enero de 2014-octubre de 2015, informó que la incidencia de ERC, alcanzó una mediana de 1,77 , pero la incidencia fue variable con un intervalo entre 0,0 y $8,68 \times 1.000$ pacientes-día ${ }^{7}$.

Antes de 2015, los regímenes de tratamiento disponibles para las infecciones por ERC consistían en polimixinas (colistina o polimixina B) con o sin agentes adicionales como carbapenémicos, tigeciclina y aminoglucósidos. Sin embargo, estos regímenes han demostrado limitada eficacia y altas tasas de nefrotoxicidad ${ }^{8}$.

Por el conocimiento actual de la morbilidad y la mortalidad asociadas con los agentes patógenos altamente resistentes y la consiguiente acción de las sociedades profesionales, la industria farmacéutica, junto con los líderes nacionales y mundiales, han llevado a un renacimiento en el pipeline de antimicrobianos ${ }^{9}$.

Se ha reportado una fuerte asociación entre las infecciones por microorganismos multi-resistentes y el incremento de costos directos, la estancia hospitalaria prolongada y el aumento de la mortalidad ${ }^{10}$. Adicionalmente, se ha descrito que existen varios elementos a ser considerados para la estimación del impacto económico de la resistencia a los fármacos antimicrobianos, tales como la mortalidad, enfermedad, tiempo y costo del tratamiento, costo del diagnóstico e impacto del no tratamiento, entre otros $^{11}$.

La combinación antimicrobiana de ceftazidima y avibactam $(\mathrm{C} / \mathrm{A})$ consiste en una cefalosporina de tercera generación y un nuevo inhibidor sintético de $\beta$-lactamasas, aprobado en 2015 por la Administración de Drogas y Alimentos (FDA, por sus siglas en inglés) de E.U.A. para el tratamiento de infecciones intra-abdominales complicadas e infecciones urinarias complicadas ${ }^{12} \mathrm{y}$, posteriormente, en 2018 para neumonía adquirida en el hospital (incluida la neumonía asociada al ventilador mecánico) ${ }^{13}$. En 2016 , recibió la autorización de comercialización de la Agencia Europea de Medicamentos (EMA, por sus siglas en inglés) para las mismas indicaciones, $\mathrm{y}$, en general, para infecciones por microorganismos aerobios gramnegativos en pacientes con opciones limitadas de tratamiento ${ }^{12}$.

Ceftazidima/avibactam, con registro sanitario en Chile desde julio de 201914, y aprobación por el Instituto de Salud Pública de las cuatro indicaciones de la EMA, es una combinación novel donde avibactam tiene actividad contra las enzimas $\beta$-lactamasas de la clase A y la clase C según Ambler, incluidas las enzimas $\beta$-lactamasas de espectro extendido (BLEEs), las carbapenemasas KPC y las enzimas $\mathrm{AmpC}$; y también sobre la carbapenemasa OXA-48 de la clase $\mathrm{D}^{15}$.

En experiencias de la vida real, se informan altas tasas de respuesta favorable al tratamiento con $\mathrm{C} / \mathrm{A}$ en pacientes con infecciones debidas a Enterobacteriaceae resistentes a carbapenémicos (ERC), con una tasa de éxito global de aproximadamente $70 \%{ }^{16}$

Estudios observacionales recientes del tratamiento de ERC (entre ellas, Klebsiella pneumoniae productora de carbapenemasa tipo KPC) han demostrado tasas más altas de curación clínica, menor riesgo de mortalidad y menor riesgo de nefrotoxicidad con la terapia basada en C/A en comparación con la terapia basada en colistin $(\mathrm{COL})^{8}$, por lo que $\mathrm{C} / \mathrm{A}$ puede ser una alternativa razonable al COL en el tratamiento de infecciones por ERC productoras de carbapenemasa tipo $\mathrm{KPC}^{6}$.

La Organización Mundial de la Salud (OMS), recomendó el año 2019 la inclusión de C/A en el Listado de Medicamentos Esenciales junto a meropenem + vaborbactam y plazomicina, recientemente registrados para el tratamiento de infecciones resistentes a múltiples medicamentos causadas por patógenos clasificados como "Prioridad crítica" en la Lista de Patógenos Prioritarios de la OMS, y clasificados bajo AWaRe como antimicrobianos de reserva ${ }^{17}$.

Hasta la fecha, no se ha realizado un análisis de costoefectividad para determinar el valor económico para la salud de C/A en comparación con el de la terapia alter- 
nativa utilizada en el contexto local. El objetivo de esta evaluación fue estimar la costo-efectividad de C/A versus colistin + meropenem $(\mathrm{COL}+\mathrm{MER})$ en el tratamiento de infecciones por ERC en Chile.

\section{Material y Métodos}

Se condujo un análisis de costo-efectividad para comparar el uso de C/A con COL+MER. El análisis se llevó a cabo con la perspectiva del pagador público y consideró costos directos asociados a los comparadores, medicamentos concomitantes, parámetros de laboratorio, hospitalización y falla renal como evento adverso asociado.

Para modelar los resultados, se utilizó un modelo de costo-efectividad desarrollado en Microsoft Excel y previamente publicado ${ }^{18}$. El modelo es un árbol de decisión (Figura 1) con un horizonte de 30 días, considerando que los datos de eficacia y mortalidad se basan en un estudio observacional que describe el seguimiento de un grupo de pacientes durante este periodo de tiempo ${ }^{6}, \mathrm{y}$ extrapolación de los desenlaces a la expectativa de vida para capturar el impacto en la cantidad y calidad de vida.

La información clínica (Tabla 1) se derivó de la literatura médica y del estudio observacional CRACKLE $^{6}$ (por su nombre en inglés Consortium on Resistance Against Carbapenems in Klebsiella and other Enterobacteriaceae); dicho estudio, considera tres desenlaces de eficacia: paciente vivo con alta domiciliaria, paciente vivo sin alta domiciliaria y paciente con muerte intrahospitalaria; se modeló la probabilidad de transición durante los 30 días observados, en función de la cohorte reportada para cada uno de los desenlaces.

El estudio CRACKLE describe el comportamiento de una cohorte de pacientes tratados con C/A o COL para infecciones por ERC durante 30 días. Para efectos de este modelo se utilizó como comparador para C/A la terapia combinada COL+MER, dado que es la que se utiliza con mayor frecuencia en el entorno local; se utilizaron los datos del estudio CRACKLE para la combinación COL+MER al verificar que ésta no es superior a la monoterapia con colistin ${ }^{19}$.

Se estimó que $94 \%$ de los pacientes con COL+MER y $63 \%$ de los pacientes con C/A requiere terapia concomitante con otros antimicrobianos, según lo reportado en el estudio CRACKLE ${ }^{6}$, utilizando como supuesto que la terapia concomitante utilizada en Chile es principalmente amikacina y tigeciclina para la estimación de costos; la no respuesta clínica descrita en el árbol de decisión corresponde a este grupo de pacientes. Adicionalmente, se definió como supuesto, que $80 \%$ de los pacientes requiere estancia hospitalaria en Unidad de Cuidados Intensivos (UCI) mientras que $20 \%$ restante puede pasar a una estancia en sala general (médica o quirúrgica).

La edad de inicio de la cohorte hipotética de pacientes fue 61 años $(50-73)^{6}$, se utilizó una tasa de descuento anual del 3\% (0-6\%), según lo recomendado por guías locales de evaluaciones económicas ${ }^{20}$, aplicada del año 2 en adelante para los desenlaces.

Los resultados se expresan como razón de costoefectividad incremental (RCEI) por Año de Vida Ganado (AVG) y por Año de Vida Ajustado por Calidad (AVAC) en pesos chilenos de 2020 y se convirtieron a dólares estadounidenses (US\$), según la tasa de cambio promedio de 2020 (US\$ 1,00 $=\$ 792,2218)^{21}$. Se considera como costo-efectiva una intervención que genere un AVAC con un costo de hasta un Producto Interno Bruto (PIB) per cápita de Chile $^{20}$.

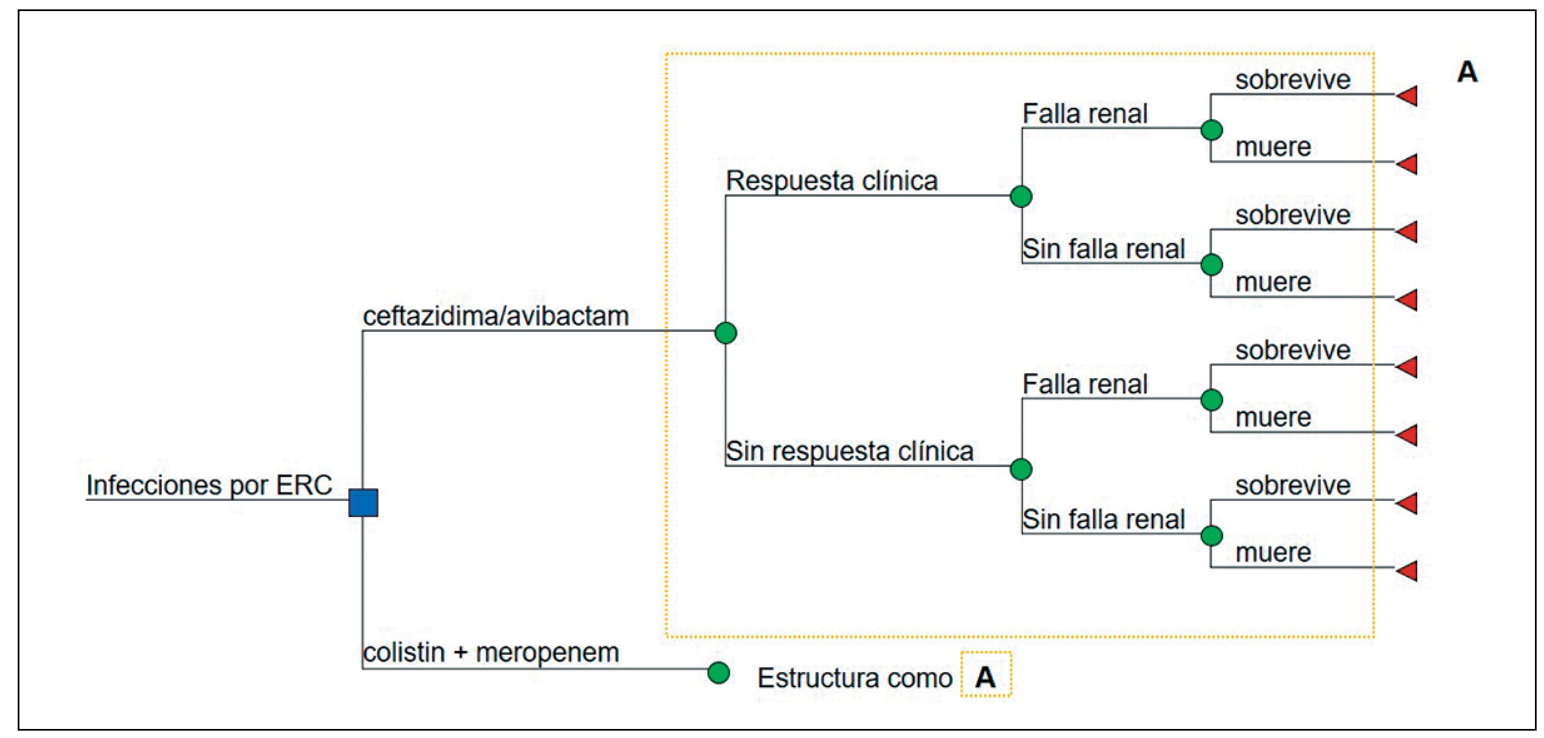

Rev Chilena Infectol 2021; 38 (1): 7-14
Figura 1. Esquema del árbol de decisión. Ceftazidima/ avibactam: 2 g/0,5 g IV c/8 $h^{4}$. Colistin: 9 millones de UI (dosis de carga), seguido de 3 millones UI c/ 8 h o 4,5 millones de UI c/12 h, IV ${ }^{8}$. Meropenem: $2 \mathrm{~g}$ IV $\mathrm{c} / 8 \mathrm{~h}$ (infusión extendida) ${ }^{8}$. 


\begin{tabular}{|c|c|c|c|c|}
\hline Variable (unidad de medida) & Media & Mínimo & Máximo & Fuente \\
\hline$\%$ pacientes con falla renal C/A & $5,0 \%$ & $3,0 \%$ & $19 \%$ & Van Duin ${ }^{6}$ \\
\hline$\%$ pacientes con falla renal COL+MER & $33,9 \%$ & $27,1 \%$ & $40,7 \%$ & Arancibia ${ }^{28}$ \\
\hline$\%$ de pacientes que requieren diálisis & $57,0 \%$ & & & Paul $^{19}$ \\
\hline Tiempo promedio de tratamiento (días) & 10,0 & 4,0 & 26,0 & Van Duin 6 \\
\hline Tiempo de falla (días) & 2,5 & 2,0 & 3,0 & Bassetti M²9 \\
\hline Utilidad en pacientes respuesta & 0,92 & & & Song $Y^{30}$ \\
\hline Utilidad en pacientes no respuesta & 0,64 & 0,54 & 0,74 & Lapointe-Shaw ${ }^{31}$ \\
\hline RR de muerte por sepsis & 0,51 & 0,49 & 0,59 & Varon $^{18}$ \\
\hline Dosis de carga de colistin al día 1 (UI) & 15.000 .000 & 12.000 .000 & 20.000 .000 & Paul $^{19}$. Van Duin 6 . Garonzik ${ }^{32}$ \\
\hline Dosis diaria de mantenimiento de colistin (UI) & 9.000 .000 & 9.000 .000 & 15.000 .000 & Paull $^{19}$. Van Duin ${ }^{6}$. Garonzik ${ }^{32}$ \\
\hline Dosis diaria de meropenem (mg) & 6 & & & Paul ${ }^{19}$ \\
\hline Dosis diaria de ceftazidima/avibactam (mg) & $6 \mathrm{~g} / 1,5 \mathrm{~g}$ & & & Folleto de producto ${ }^{15}$ \\
\hline Dosis diaria de tigeciclina (mg) & 100 & & & Supuesto \\
\hline Dosis diaria de amikacina (mg) & 900 & & & Supuesto \\
\hline
\end{tabular}

\begin{tabular}{|c|c|c|c|c|c|}
\hline \multirow{2}{*}{$\begin{array}{l}\text { Variable } \\
\text { Meropenem vial } 1 \mathrm{~g}\end{array}$} & Valor promedio & Mínimo & \multicolumn{2}{|c|}{ Máximo } & \multirow{2}{*}{$\begin{array}{c}\text { Fuente } \\
\text { Mercado Público }\end{array}$} \\
\hline & $\$ 5.400$ & $\$ 2.000$ & $\$$ & 8.000 & \\
\hline Colistin 3 millones UI & $\$ 10.480$ & $\$ 9.800$ & $\$$ & 23.000 & Mercado Público \\
\hline Ceftazidima/avibactam vial & $\$ 87.360$ & $\$ 78.624$ & $\$$ & 96.096 & Precio de lista \\
\hline Tigeciclina 50 mg & $\$ 22.928$ & $\$ 11.900$ & $\$$ & 38.693 & Mercado Público \\
\hline Amikacina 500 mg & $\$ \quad 208$ & $\$ \quad 194$ & $\$$ & 380 & Mercado Público \\
\hline Día cama en el piso & $\$ 39.430$ & $\$ 31.544$ & $\$$ & 47.316 & Tarifas MAl \\
\hline Día cama en UCl & $\$ 163.910$ & $\$ 131.128$ & $\$$ & 196.692 & Tarifas MAI \\
\hline Prevención de insuficiencia renal & $\$ 57.290$ & $\$ 45.832$ & $\$$ & 68.748 & EVC \\
\hline Diálisis mensual & $\$ 856.370$ & $\$ 685.096$ & & 027.644 & Tarifas MAl \\
\hline Creatininemia & $\$ 16.130$ & $\$ 14.517$ & $\$$ & 17.743 & Tarifas MAI \\
\hline Nitrógeno ureico & $\$ \quad 1.180$ & $\$ \quad 1.062$ & $\$$ & 1.298 & Tarifas MAI \\
\hline Hemograma & $\$ \quad 2.680$ & $\$ \quad 2.412$ & $\$$ & 2.948 & Tarifas MAI \\
\hline Electrolitos plasmáticos & $\$ \quad 1.100$ & 990 & $\$$ & 1.210 & Tarifas MAI \\
\hline
\end{tabular}

\begin{tabular}{|c|c|c|c|c|c|c|c|c|}
\hline & Costo & $\Delta$ Costo & AVAC* & $\triangle$ AVAC & AVG* & $\Delta$ AVG & RCEI \$/AVAC & RCEI \$/AVG \\
\hline $\mathrm{COL}+\mathrm{MER}$ & $\$ 4.380 .118$ & & 4,48 & & 6,80 & & & \\
\hline C/A & $\$ 6.523 .572$ & $\$ 2.143 .454$ & 6,76 & 2,28 & 9,08 & 2,28 & $\$ 938.715$ & $\$ 940.488$ \\
\hline
\end{tabular}


Para la estimación de desenlaces clínicos se realizaron las simulaciones a 30 días asumiendo un comportamiento similar al observado en el estudio CRACKLE ${ }^{6}$; para estimar los AVG se considera la proporción de pacientes que permanece viva en dicho horizonte, extrapolando a la expectativa de vida de Chile: 80,6 años, según lo reportado por organismos oficiales ${ }^{22}$. La extrapolación de calidad de vida y supervivencia a largo plazo se realiza a partir de literatura científica sobre sepsis, debido a la limitación de datos específicos de ERC. Las utilidades para estimar los AVAC a partir de los AVG se tomaron de la literatura científica (Tabla 1).

Para verificar la robustez del modelo se realizaron análisis de sensibilidad ${ }^{23}$ determinístico, univariado, que consiste en modificar sólo el valor de un parámetro a la vez y ver cómo afecta al resultado, y probabilístico mediante una simulación de Montecarlo con 5.000 iteraciones, en las que se generaron diferentes escenarios, resultado de las combinaciones de los posibles valores de las variables según su distribución de probabilidad, uniforme o beta para los datos clínicos y gamma para los costos.

\section{Costos}

Los costos fueron tomados de reportes oficiales de Chile $^{24-26}$ (Tabla 2) y actualizados a valores 2020; para aquellos valores reportados en 2019 o en años previos, se aplicó la inflación reportada por el Banco Central ${ }^{27}$.

Los costos de exámenes de laboratorio se estimaron a partir de validaciones con profesionales de la salud locales, en que se definió como supuesto que se requiere realizar un hemograma cada $48 \mathrm{~h}$ para ambos comparadores y exámenes de creatinina, nitrógeno ureico y electrolitos cada $24 \mathrm{~h}$ para COL+MER y cada $72 \mathrm{~h}$ para C/A.

Para la información de los medicamentos, se utilizó el precio promedio y como distribución el precio mínimo y máximo reportados entre enero y marzo de 2020 en el portal de compras públicas de Chile, ChileCompra ${ }^{25}$, que corresponde a la plataforma de la Dirección de Compras y Contratación Pública de Chile dependiente del Ministerio de Hacienda. Para las otras variables de costos en que sólo se reporta un dato promedio, se utilizó como mínimo el valor reportado menos $10 \%$ y para el valor máximo el valor reportado más $10 \%$.

\section{Resultados}

Bajo los supuestos del modelo, C/A se asoció con una menor tasa de mortalidad $(9,2 \%)$ y menor uso de terapias antimicrobianas adicionales $(63,0 \%)$ en comparación con COL+MER (32,0 y 94,0\%, respectivamente), resultando en un incremento de 2,28 AVAC por paciente en comparación con $\mathrm{COL}+\mathrm{MER}$.

Los costos incrementales de $\mathrm{C} / \mathrm{A}$ en comparación con COL+MER, se deben parcialmente a la baja tasa de mortalidad observada en pacientes que recibieron esta primera terapia.

La RCEI estimada para C/A vs COL+MER fue $\$ 940.488$ (US\$1.187,2) por AVG y $\$ 938.715$ (US\$1.184,9) por AVAC (Tabla 3). Con una disponibilidad a pagar de 1 PIB per cápita (estimado en $\$ 10.464 .365$ para 2019 [US\$13.208,9]) por AVAC ganado, de acuerdo con lo recomendado en la Guía Metodológica para la evaluación económica de intervenciones en salud en $\mathrm{Chile}^{20}$, $\mathrm{C} / \mathrm{A}$ sería una alternativa costo-efectiva en comparación con COL+MER.

\section{Análisis de sensibilidad}

El análisis de sensibilidad determinístico representado en el diagrama de tornado, indicó que el ICER es altamente sensible a las variaciones de tiempo de tratamiento, seguido por las variables de utilidad de los pacientes que responden a monoterapia y costo de las terapias en comparación, principalmente (Figura 2).

El análisis de sensibilidad probabilístico arrojó que este resultado se mantendría en $100 \%$ de las simulaciones, es decir, las 5.000 iteraciones realizadas ubican la RCEI de C/A vs COL+MER por debajo del umbral de disposición a pagar de 1 PIB per cápita por AVAC, lo que se refleja a través de un diagrama de dispersión de costos incrementales versus efectividad incremental (Figura 3).

\section{Discusión}

Al no contar con ensayos clínicos para la indicación de $\mathrm{C} / \mathrm{A}$ en infecciones por microorganismos aerobios gramnegativos en pacientes con opciones limitadas de tratamiento y a los escasos datos locales, la transición de los pacientes por los estados de salud se basa en un estudio observacional cuyo horizonte es de 30 días $^{6}$. La menor tasa de mortalidad de C/A impacta en los costos de hospitalización, pues una mayor proporción de pacientes tratados con C/A permanecen con vida más tiempo que aquellos que utilizan COL, por lo que se requiere mayor uso de recursos sanitarios.

En el modelo planteado, sólo se modeló la falla renal como potencial evento adverso asociado a las terapias en comparación, pero no se consideraron las potenciales des-utilidades derivadas de ésta. Sería relevante realizar un análisis más ajustado a la realidad local en cuanto a los tiempos de hospitalización, a los gastos asociados a los tratamientos y a los eventos que se relacionen con éstos, por lo que se requiere que la práctica local se registre y se analice para tener insumos que soporten los supuestos a utilizar en un modelo económico.

Los recursos económicos para financiar la atención sanitaria son limitados, lo que dificulta la elección cuando existe más de una alternativa disponible; los resultados 


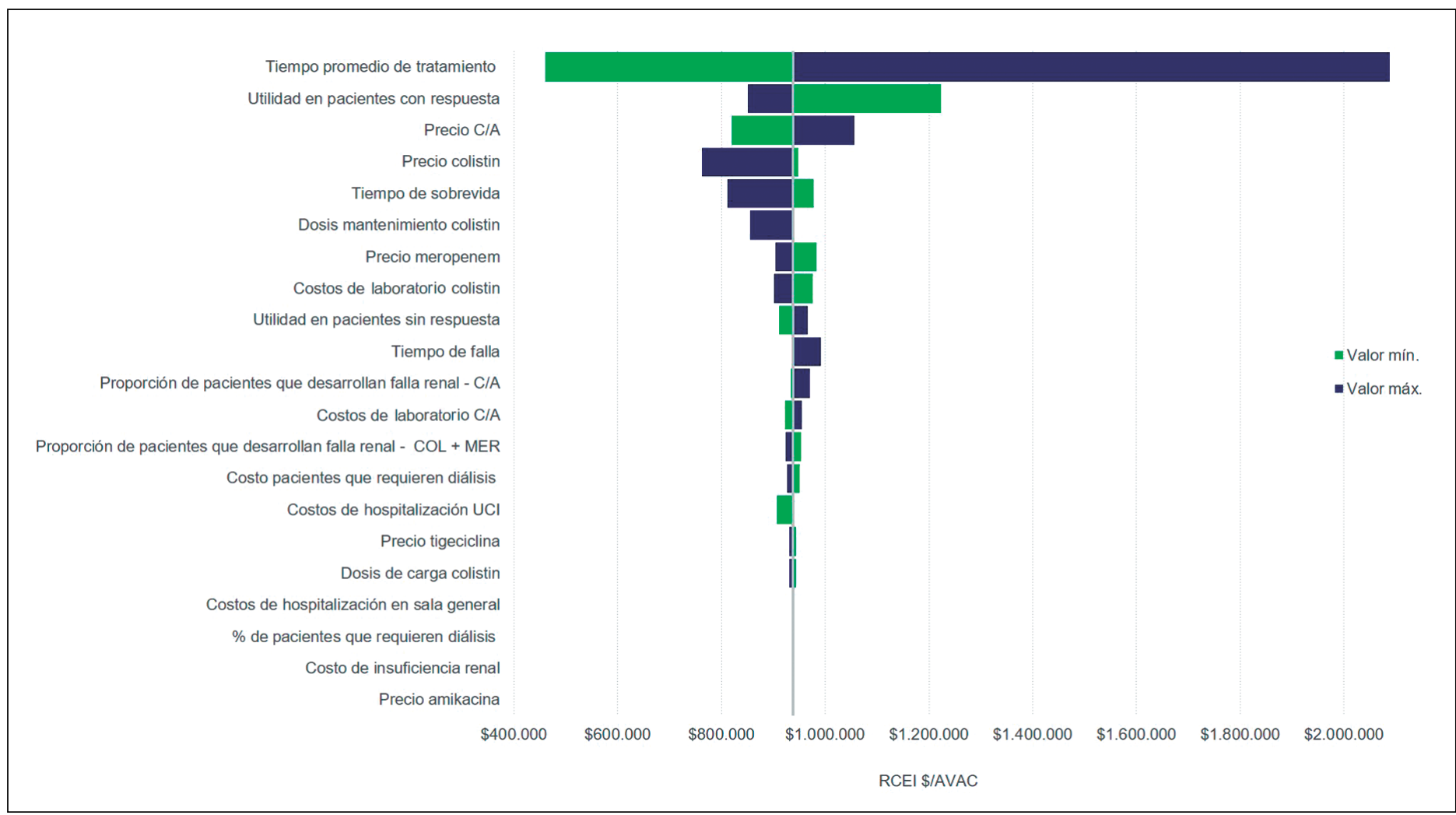

Figura 2. Diagrama de tornado del análisis de sensibilidad determinístico.

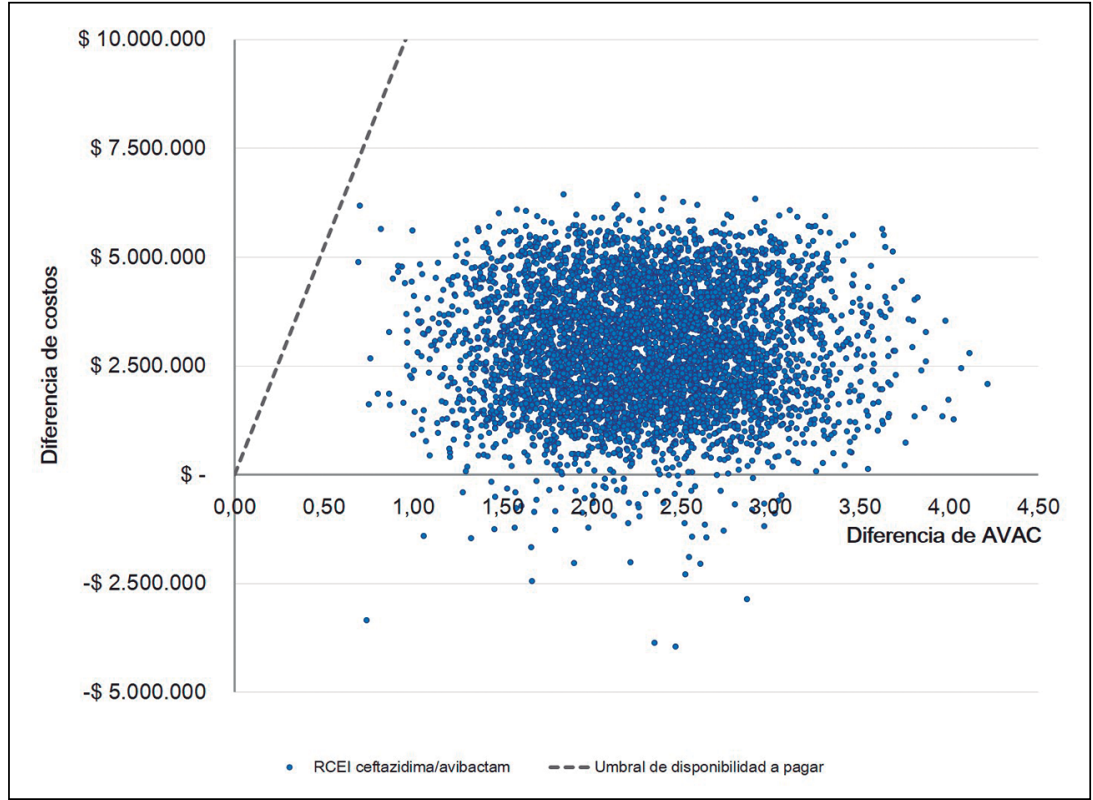

Figura 3. Gráfico de dispersión. Las 5.000 iteraciones de la simulación se encuentran por debajo del umbral de disposición a pagar estimado para Chile (1 PIB per cápita por AVAC ganado), indicando costo-efectividad de C/A vs COL+MER. de los análisis de costo-efectividad son una herramienta valiosa para evaluar alternativas de intervención de salud que en ocasiones parecen tener costos de adquisición sustancialmente diferentes, pero que con una mirada más holística pueden demostrar que el costo inicial se justifica por los desenlaces clínicos que se obtienen. Cabe resaltar que estos análisis no buscan sustituir la toma de decisión del médico tratante, sino brindar información adicional para que haya una toma de decisiones informada en la que se consideren todas las variables posibles que hacen parte de la atención sanitaria.

Bajo los supuestos planteados y en el escenario analizado, C/A demostró reducir la proporción de muertes e incrementar los AVG y los AVAC para el tratamiento de infecciones por ERC en Chile, resultando en una alternativa costo-efectiva vs COL+MER.

Los resultados obtenidos coinciden con otros estudios realizados en Colombia ${ }^{18}$ y E.U.A. ${ }^{8}$, que también muestran mejores resultados clínicos con C/A para el tratamiento de infecciones por ERC, en comparación con las terapias estándar utilizadas en dichos países. La principal diferencia del modelo realizado en Colombia respecto al modelo local es que en la terapia concomitante 
se utiliza fosfomicina y tigeciclina, mientras que en el contexto local se utilizan amikacina y tigeciclina; las demás características son similares, dado que éste fue el modelo utilizado como referencia para el modelo local; la RCEI estimada para C/A vs COL+MER en Colombia fue de \$2.076 dólares americanos por AVAC. En el caso del modelo estadounidense, éste fue realizado para bacteriemia y neumonía por enterobacterias resistentes a carbapenémicos; con un horizonte de tiempo de 5 años se desarrolló un modelo tipo Markov con ciclos anuales en que se comparó C/A vs COL. El estudio reporta que con base en los estándares de disponibilidad a pagar del país
(\$100.000 a \$150.000 dólares americanos por AVAC), $\mathrm{C} / \mathrm{A}$ tendría una RCEI aceptable vs. COL (\$95.000 dólares americanos por AVAC).

Cabe resaltar que el estudio CRACKLE fue fuente de información para algunas variables clínicas, lo que confirma la falta de ensayos clínicos para infecciones por microorganismos aerobios gramnegativos en pacientes con opciones limitadas de tratamiento.

Agradecimientos. A la Dra. María Paz Ramos por su apoyo en la recolección de los datos locales utilizados como insumo para el modelo económico.

\section{Referencias bibliográficas}

1.- Gordon J, Darlington O, McEwan P, Lumley M, Taie A, Hicks M, et al. Estimating the value of new antimicrobials in the context of antimicrobial resistance: development and application of a dynamic disease transmission model [published online ahead of print, 2020 Apr 6]. Pharmacoeconomics 2020:10.1007/ s40273-020-00906-6. doi:10.1007/s40273-02000906-6

2.- Ramos-Castañeda J A, Ruano-Ravina A, Barbosa-Lorenzo R, Paillier-Gonzalez J E, Saldaña-Campos J C, Salinas D F, et al. Mortality due to KPC carbapenemaseproducing Klebsiella pneumoniae infections: Systematic review and meta-analysis: Mortality due to KPC Klebsiella pneumoniae infections. J Infect 2018; 76 (5): 438-48. doi:10.1016/j. jinf.2018.02.007.

3.- O'Neill J. Tackling drug-resistant infections Globally: Final report and recommendations. The Review on Antimicrobial Resistance.; 2016. https://amr-review.org/sites/default/ files/160518_Final paper_with cover.pdf.

4.- National Center for Emerging and Zoonotic Infectious Diseases/ Centers for Disease Control and Prevention (CDC). Facility guidance for control of carbapenem-resistant Enterobacteriaceae (CRE). 2015. https://www. cdc.gov/hai/pdfs/cre/CRE-guidance-508.pdf.

5.- Livermore D M. Has the era of untreatable infections arrived? J Antimicrob Chemother 2009; 64 Suppl 1: i29-i36. doi:10.1093/jac/ dkp255.

6.- van Duin D, Lok J J, Earley M, Cober E, Richter S S, Pérez F, et al. Colistin versus ceftazidime-avibactam in the treatment of infections due to carbapenem-resistant Enterobacteriaceae. Clin Infect Dis 2018; 66 (2): 163-71. doi:10.1093/cid/cix783.

7.- Acuña M P, Cifuentes M, Silva F, Rojas Á, Cerda J, Labarca J. Incidence of multi-resistant bacteria in Intensive Care Units of Chilean hospitals. Rev Chil Infectol 2017; 34 (6):
570-5. doi:10.4067/S071610182017000600570.

8.- Simon M, Sfeir M, Calfee D, Satlin M. Cost-effectiveness of ceftazidime-avibactam for treatment of carbapenem-resistant Enterobacteriaceae bacteremia and pneumonia. Antimicrob Agents Chemother 2019; 63 (12): e00897-19. doi: 10.1128/AAC.00897-19.

9.- Strich J R, Ricotta E, Warner S, Lai Y L, Demirkale C Y, Hohmann S F, et al. Pharmacoepidemiology of ceftazidimeavibactam use: a retrospective cohort analysis of 210 US hospitals. Clin Infect Dis 2020: ciaa061. doi: 10.1093/cid/ciaa061.

10.- Serra-Burriel M, Keys M, Campillo-Artero C, Agodi A, Barchitta M, Gikas A, et al. Impact of multi-drug resistant bacteria on economic and clinical outcomes of healthcare-associated infections in adults: Systematic review and meta-analysis. PLoS One 2020; 15 (1): e0227139. doi: 10.1371/journal.pone.0227139.

11.- McGowan J J. Economic impact of antimicrobial resistance. Erratum in: Emerg Infect Dis 2001; 7 (4): 765 . Emerg Infect Dis 2001; 7 (2): 286-92. doi:10.3201/ eid0702.010228

12.- Tumbarello M, Trecarichi E M, Corona A, De Rosa F G, Bassetti M, Mussini C, et al. Efficacy of ceftazidime-avibactam salvage therapy in patients with infections caused by Klebsiella pneumoniae carbapenemase-producing $K$. pneumoniae. Clin Infect Dis 2019; 68 (3): 355 64. doi:10.1093/cid/ciy492.

13.- FDA approves new treatment for hospitalacquired and ventilator-associated bacterial pneumonia. https://www.fda.gov/news-events/ press-announcements/fda-approves-newtreatment-hospital-acquired-and-ventilatorassociated-bacterial-pneumonia.

14.- Registro sanitario de Zavicefta. http:// registrosanitario.ispch.gob.cl/Ficha. aspx?RegistroISP=F-24968/19.

15.- Basado en el Folleto de Información al Profesional vigente de Zavicefta ${ }^{\circledR}$ (cetfazidima/ avibactam) disponible en www.pfizerpro.cl.
16.- Vena A, Giacobbe D R, Castaldo N, Cattelan A, Mussini C, Luzzati R, et al. Clinical experience with ceftazidime-avibactam for the treatment of infections due to multidrug-resistant gram-negative bacteria other than carbapenemresistant Enterobacterales. Antibiot 2020; 9 (2) 71. doi:10.3390/antibiotics 9020071 .

17.- World Health Organization. The Selection and use of essential medicines Report of the 22nd WHO Expert Committee on the 2019. Selection and use of essential medicines. WHO Headquarters, Geneva 1-5 April 2019. https://apps.who.int/iris/bitstream/ handle/10665/325773/WHO-MVP-EMP-IAU2019.05-eng.pdf?ua $=1$.

18.- Varon F A, Castaño Gamboa N, Reyes Sánchez J M, Lemos E V. Cost-effectiveness analysis of ceftazidime-avibactam versus colistinmeropenem in the treatment of infections due to carbapenem-resistant enterobacteriaceae in Colombia. Value Heal 2019; 22 (3): S650. doi:https://doi.org/10.1016/j.jval.2019.09.1307.

19.- Paul M, Daikos G L, Durante-Mangoni E, Yahav D, Carmeli Y, Benattar Y D, et al. Colistin alone versus colistin plus meropenem for treatment of severe infections caused by carbapenem-resistant Gram-negative bacteria: an open-label, randomised controlled trial. Lancet Infect Dis 2018; 18 (4): 391-400. doi:10.1016/S1473-3099(18)30099-9.

20.- Ministerio de Salud de Chile. Guía Metodológica para la evaluación económica de intervenciones en salud en Chile. https://desal. minsal.cl/wp-content/uploads/2013/09/EE FINAL_web.pdf. Published 2013. Accessed April 7, 2020.

21.- Banco Central de Chile. Dólar Observado 2020. https://si3.bcentral.cl/indicadoressiete/secure/ IndicadoresDiarios.aspx. Accessed January 18, 2021.

22.- Instituto Nacional de Estadísticas (INE). Esperanza de vida al nacer. https://www.ine.cl/ inicio. Accessed April 13, 2020.

23.- Ferriols R, Alós M. Métodos de análisis de la incertidumbre [Methods for analyzing 
uncertainty]. Farm Hosp 2011; 35 Suppl 2:3-9. doi:10.1016/S1130-6343(11)70016-2.

24.- FONASA. Modalidad de Atención Institucional. Planilla MAI 2020. https://www. fonasa.cl/sites/fonasa/prestadores/modalidadatencion-institucional.

25.- Mercado Público. ChileCompra. https://www. mercadopublico.cl/Home.

26.- Instituto de Administración en Salud. Estudio de Verificación del Costo Esperado Individual Promedio por Beneficiario del Conjunto Priorizado de Problemas de Salud con Garantías Explícitas 2018.; 2018. http:// www.ias.uchile.cl/investigacion/estudio-deverificacion-del-costo-esperado-individualpromedio-por-beneficiario-del-conjuntopriorizado-de-problemas-de-salud-congarantias-explicitas-2018.
27.- Banco Central de Chile. Indice de Precios al Consumidor (IPC). https://si3.bcentral.cl/ Indicadoressiete/secure/Indicadoresdiarios. aspx. Accessed April 13, 2020

28.- Arancibia J M, Bravo F, Muñoz A. Incidencia $\mathrm{y}$ factores de riesgo de nefrotoxicidad en pacientes tratados con colistín en UCI. XXXII Congreso Chileno de Infectología. http:// www.sochinf.cl/portal/templates/sochinf2008/ documentos/2015/Libro_congreso_2015.pdf. Published 2015. Accessed April 6, 2020.

29.- Bassetti M, Montero J G, Paiva J A. When antibiotic treatment fails. Intensive Care Med 2018; 44 (1): 73-5. doi: 10.1007/s00134-0174962-2.

30.- Song Y, Tai J H, Bartsch S M, Zimmerman R K, Muder R R, Lee B Y. The potential economic value of a Staphylococcus aureus vaccine among hemodialysis patients. Vaccine 2012; 30 (24): 3675-82. doi:10.1016/j. vaccine.2012.03.031.

31.- Lapointe-Shaw L, Voruganti T, Kohler P, Thein H, Sander B, McGeer A. Costeffectiveness analysis of universal screening for carbapenemase-producing Enterobacteriaceae in hospital inpatients. Eur J Clin Microbiol Infect Dis 2017; 36 (6): 1047-55. doi:10.1007/ s10096-016-2890-7.

32.- Garonzik S M, Li J, Thamlikitkul V, Paterson D L, Shoham S, Jacob J, et al. Population pharmacokinetics of colistin methanesulfonate and formed colistin in critically ill patients from a multicenter study provide dosing suggestions for various categories of patients. Antimicrob Agents Chemother 2011; 55 (7): 3284-94. doi:10.1128/AAC.01733-10. 